\title{
PERCEPÇÕES E REPRESENTAÇÕES SOCIAIS DE PROFESSORES ACERCA DE AVALIAÇÃO DA APRENDIZAGEM: UM ESTUDO DAS PRODUÇÕES EM TESES E DISSERTAÇÕES (2009-2019) ${ }^{1}$
}

http://dx.doi.org/10.5902/2318133855396

\author{
Diego Mota ${ }^{2}$ \\ Maria Apparecida Campos Mamede-Neves ${ }^{3}$
}

\begin{abstract}
Resumo
O estudo teve por objetivo mapear a produção sobre avaliação com foco nas percepções e representações dos professores da educação básica. Para isso foram analisadas dissertações e teses disponibilizadas na BDTD, publicadas entre o período 2009-2019. Observou-se a hegemonia de uma perspectiva teórica construtivista de avaliação formativa direcionando o debate acadêmico. Predominam, no campo analítico, pesquisas qualitativas e estudos de caso que convergem, em suas conclusões, para a constatação de um pensamento social cujas percepções se aproximam de uma pedagogia avaliativa tradicional, destacando as dificuldades dos professores de incorporar os ideais pedagógicos contemporâneos.

Palavras-chave: avaliação da aprendizagem; estudo de revisão; representações sociais.
\end{abstract}

\section{PERCEPTIONS AND SOCIAL REPRESENTATIONS OF TEACHERS ABOUT CLASSROOM ASSESSMENT: A STUDY OF REVISION IN BRAZILIAN MASTER'S THESES AND DOCTORAL DISSERTATIONS (2009-2019)}

\begin{abstract}
This essay aims analyses the academic production on classroom assessment with a focus on the perceptions and representations of basic education Brazilian teachers. For this, doctoral dissertations and master's theses made available at digital platform BDTD, published between 2009-2019, were analyzed. The hegemony of a constructivist theoretical perspective of assessment for learning was observed, directing the academic debate. In the analytical field, qualitative research and case studies predominate that converge in their conclusions for the verification of a social thought whose perceptions are close to a traditional summative assessment, highlighting the difficulties of teachers to incorporate in them practices the proposals contemporary of assessment as learning.

Keywords: classroom assessment; review study; social representations.
\end{abstract}

\footnotetext{
${ }^{1} \mathrm{O}$ presente trabalho foi realizado com apoio da Capes, código de financiamento 001.

2 Colégio Pedro II, Brasil. E-mail: diegoomota@gmail.com.

3 Pontifícia Universidade Católica do Rio de Janeiro, Brasil. E-mail: apmamede@gmail.com.
} 


\section{Introdução}

tema avaliação é uma questão substancial nas pesquisas educacionais por estar diretamente relacionado aos diversos aspectos que potencializam as aprendizagens dos estudantes. Por esse motivo, mais do que um objeto clássico, esse é um campo de análise que carece de contínua e permanente investigação acadêmica, especificamente em países como o Brasil que apresenta, ainda no século 21, a necessidade de investimentos em políticas voltadas para a educação básica e a formação de professores.

Nesse sentido, esta pesquisa busca descrever um panorama das produções acadêmicas em teses e dissertações produzidas no período de 2009-2019, sobre o tema avaliação da aprendizagem, com a finalidade de mapear os resultados e as análises realizados na última década. A partir dessa observação temos o objetivo de desenvolver um conjunto de resultados que apresente alguma nova contribuição para o campo, junto a outros estudos produzidos na contemporaneidade.

De acordo com Cresswel (2007) as revisões de literatura são ensaios que gravitam em torno de pesquisas sobre uma determinada área do conhecimento e seus resultados se fundamentam no mapeamento de estudos selecionados de acordo com sua relevância, fornecendo, dessa maneira, uma estrutura para analisar seus resultados conjuntamente. Ao realizarmos uma análise exploratória em portais de periódicos e bases de teses e dissertações, reconhecemos ser volumosa a produção acadêmica no campo da avaliação, devido à diversidade de possibilidades de investigações.

Por conta disso houve a necessidade de definir o recorte temporal e a especificação do tema. Baseado nessa lógica no estudo aborda-se as produções acadêmicas cujo objeto de análise são as percepções, as crenças e as representações sociais de professores sobre avaliação da aprendizagem no contexto da educação básica, publicadas entre 2009 e 2019.

Outros pesquisadores têm dedicado esforços para mapear o campo investigativo nas últimas décadas. Dentre os referenciais com maior proximidade cronológica, com o foco do nosso estudo, Jacomini (2014) apresenta uma revisão sistemática das produções em teses e dissertações sobre o cenário das avaliações da aprendizagem no contexto de ciclos na educação básica. A autora descreve que os resultados das pesquisas realizadas no período entre 2000 e 2010 indicam um desalinhamento entre os documentos legais e as práxis avaliativas da sala de aula. Além disso essa pesquisa ressalta que, embora 0 discurso dos professores revele percepções pouco tradicionais dos processos avaliativos, suas práticas caminham em outra direção.

Tais apontamentos também foram identificados no estudo de Costa (2010) sobre as concepções de avaliação na educação básica dentro do período de 1999 a 2008. De acordo com a autora, apesar de encontrarmos nas pesquisas discursos de professores que se aproximam das dimensões formativas, na escola "a avaliação permanece na dimensão da nota" (p. 116).

Diante dos resultados apresentados por autores como Calderón e Poltronieri (2013), Costa (2010) e Jacomini (2014) sobre o tema na década de 2000, o presente trabalho pode ser um caminho promissor para indicar possíveis mudanças ou permanências reveladas pelos estudos direcionados ao assunto. Em busca dessa linha investigativa, 
consideramos fundamental seguir um percurso metodológico que nos permita identificar e analisar as perspectivas e dimensões mais exploradas ou desconsideradas em pesquisas sobre os diferentes lugares e segmentos da educação básica.

\section{Metodologia}

Como já salientamos esta pesquisa tem por objetivo mapear a produção acadêmica sobre as percepções e representações sociais de professores acerca de avaliação da aprendizagem. Assumindo uma perspectiva de combinação metodológica mista, de acordo com Flick (2009), realizamos um estudo de revisão por meio da análise das teses e dissertações publicadas em língua portuguesa nos últimos dez anos.

O presente trabalho se estrutura no modelo proposto por David Gough e colaboradores $(2004,2018)$ para revisões de literatura. Os autores descrevem uma série de procedimentos que possibilitam a construção de um estudo objetivo em relação às etapas de busca, avaliação e síntese seguidas em estudos sistemáticos.

Embora nossa análise tenha um foco descritivo com objetivos quanti-qualitativos, a sistematização dos procedimentos adotados é fundamental para a estruturação dos trabalhos inclusos nesta investigação. Esse caminho também possibilita a continuidade cronológica da revisão, com base no detalhamento das coordenadas seguidas.

De acordo com a perspectiva de Gough e colaboradores (2012) a revisão de literatura, em todas as suas etapas, deve ser um percurso construído de maneira projetada e delineada. Os autores propõem que estudos dessa natureza sigam processos de seleção e categorização, que incluem as etapas descritas no diagrama da figura 1.

Figura 1 -

Fluxograma dos procedimentos metodológicos para estudos de revisão. Definição do objeto da pesquisa
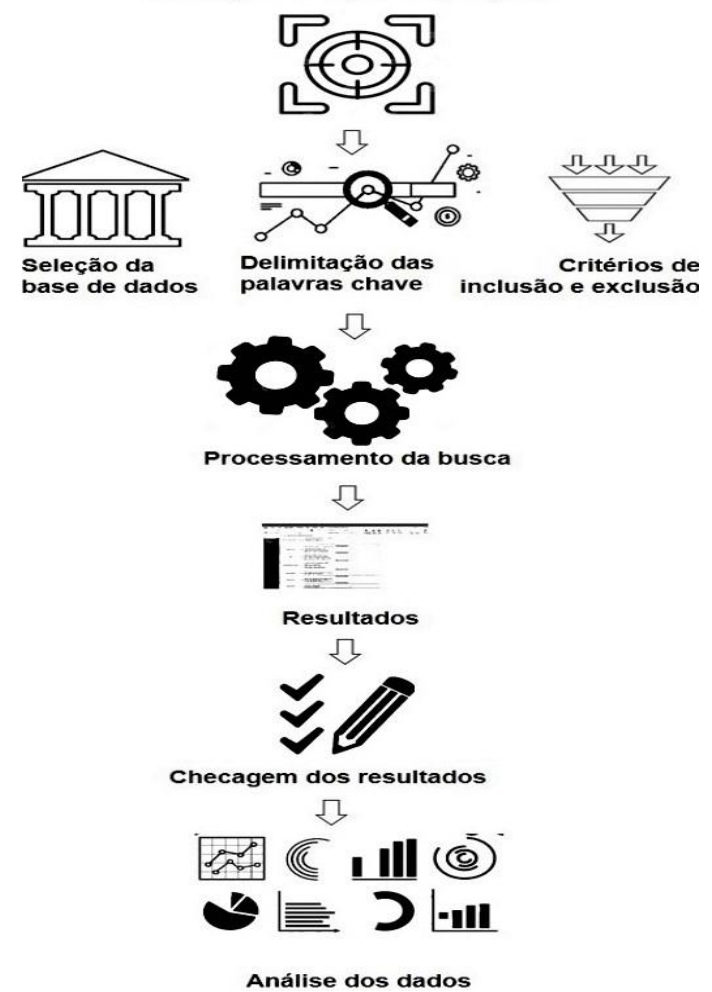

Fonte: autores com base em Gough et al., 2012. 


\section{Procedimentos metodológicos: definição do objeto de pesquisa e das bases de dados}

Procedemos as etapas propostas com a definição do objetivo do estudo: explorar as produções acadêmicas sobre as percepções e representações sociais de professores da educação básica acerca de avaliação da aprendizagem (2009-2019). Desse modo, com o recorte do universo amostral, delimitamos as palavras-chave a serem usadas em nossa busca. Para isso, utilizamos os termos "avaliação da aprendizagem" e "professores". A opção metodológica dos termos combinados de busca com o operador booleano "and" possui a finalidade de especificar os corpora textuais próximos do objeto de investigação, dada a amplitude do tema no campo educacional. Realizamos o levantamento numa base de dados - a BDTD - em maio de 2020.

\section{Demarcação das configurações de busca}

Em seguida delimitamos as configurações de pesquisa na plataforma. Utilizamos as definições de busca avançada descrita na figura 2. No portal da BDTD configuramos as seguintes especificações no refinamento: pesquisas de mestrado e doutorado, período de 2009 a 2019, apresentadas em programas de pós-graduação em Educação, na área de concentração da Educação. Encontramos 406 resultados. O encorpado volume do material selecionado está relacionado à amplitude do tema da avaliação, à centralidade do campo educacional na pós-graduação brasileira e à presença dos termos de busca de forma preeminente nas pesquisas da área.

Figura 2 -

Página de busca do catálogo BDTD após as definições dos algoritmos de busca e o resultado apresentado.

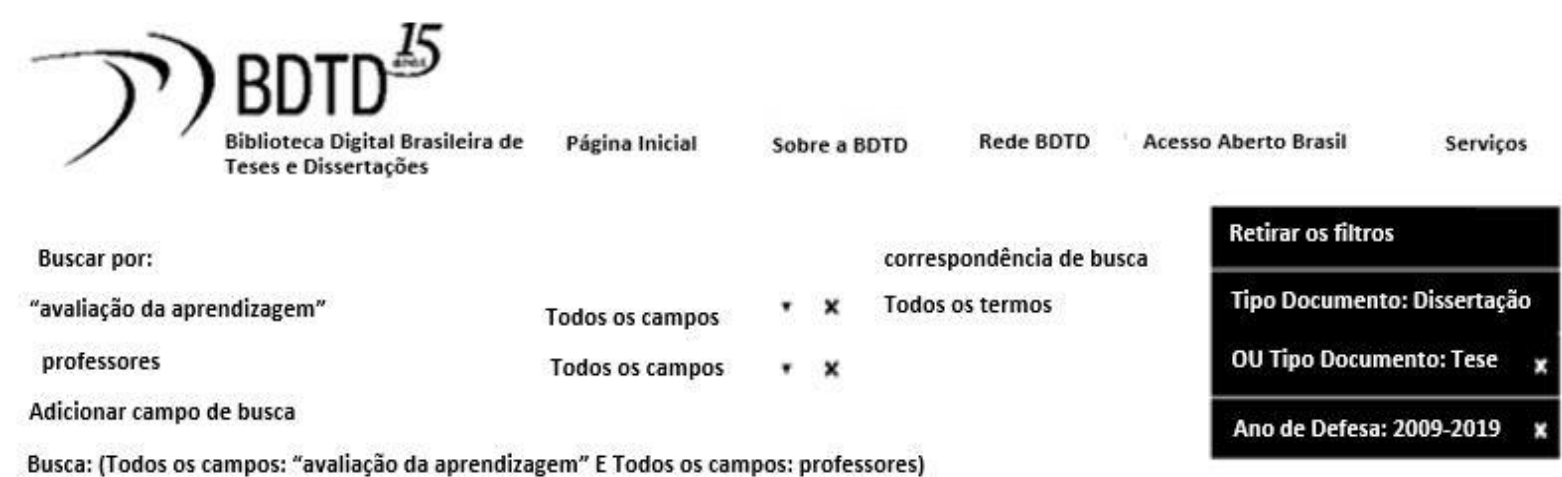

Amostrar: 1 - 20 resultados de 406 tempo de busca $0.55 \mathrm{~s} \quad$ Ordenar Data ascendente

Fonte: dados da pesquisa.

\section{Pré-seleção do material textual: aplicação dos critérios de inclusão-exclusão}

Os dados brutos revelaram uma grande quantidade de estudos para essa etapa da investigação. Analisamos as pesquisas diretamente na plataforma a partir de seus títulos, resumos e exploração dos textos, quando os dados de busca não estavam clarificados em sua descrição. Anteriormente definimos os parâmetros que limitam o universo 
amostral a ser explorado. Como critérios de inclusão consideramos pesquisas sobre a avaliação da aprendizagem, no contexto da educação básica, relacionadas às percepções e representações sociais de professores.

Como critérios de exclusão demarcamos que os estudos relacionados ao contexto da educação superior, à avaliação de professores, à avaliação de políticas educacionais, à avaliação de programas, à educação a distância, às avaliações externas, às metaanálises e à avaliação na educação infantil seriam desconsiderados, pois o foco estava fora dos propósitos de nossa investigação.

Os processos de inclusão e exclusão foram efetuados a partir de sua identificação no título, resumo ou palavras-chave do material textual analisado. Também aplicamos essas especificações de seleção na leitura dos textos. Por esse motivo desconsideramos quatro trabalhos que se distanciavam da amostra por falta de clareza em seus resumos quanto aos objetivos do estudo. Assim, das 406 ocorrências foram selecionados para a etapa consecutiva 40 dissertações e teses de doutorado.

As buscas na base de dados foram replicadas por outro pesquisador colaborador. Estas trilharam o mesmo protocolo das etapas anteriores, tendo as considerações de um terceiro pesquisador em caso de divergências quanto aos critérios de triagem do recorte amostral. Depois da concordância entre os pesquisadores, com as interseções e convergência dos resultados, iniciamos o tratamento dos dados obtidos.

\section{Leitura e categorização do corpus textual}

$\mathrm{Na}$ execução dessa etapa exportamos os resumos e arquivos obtidos na base de dados e os textos completos em suas plataformas de referência. Selecionamos um total de 40 trabalhos, os quais foram tabulados e submetidos à análise categorial temática, conforme Bardin (2011), com o auxílio do programa Excel.

Cabe salientar que todos os 40 trabalhos selecionados foram analisados pelos pesquisadores e suas informações foram tipificadas de acordo com as categorias descritas no quadro 1. Após classificarmos sistematicamente o corpus textual obtido iniciamos sua análise.

Quadro 1 -

Categorias aplicadas na análise dos arquivos, teses e dissertações selecionados para este estudo.

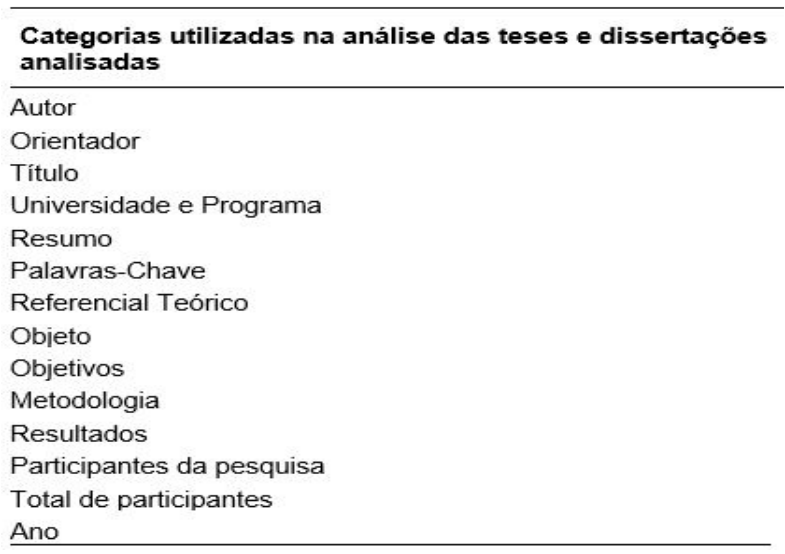

Fonte: dados da pesquisa. 


\section{Resultados}

Após a checagem da consonância dos procedimentos e do material apurado iniciamos a discussão dos resultados com o balanço da análise do conjunto de teses e dissertações. Dentre as 406 ocorrências identificadas encontramos um total de 40 estudos - 24 teses e 16 dissertações - sobre percepções e representações sociais de professores acerca de avaliação da aprendizagem na base de dados consultada. $\mathrm{Na}$ tabela 1 descreve-se o total de teses e dissertações produzidas nos distintos programas de pós-graduação.

Tabela 1 -

Total de teses e dissertações sobre percepções e representações sociais de professores acerca de avaliação da aprendizagem.

\begin{tabular}{c|c|c|c|c|c}
\hline IES & Trabalhos & IES & Trabalhos & IES & Trabalhos \\
\hline UFPB & 6 & UCG & 1 & UFMA & 1 \\
\hline UFC & 5 & UEL & 3 & UFMG & 1 \\
\hline UFPE & 3 & UNB & 1 & UFPI & 1 \\
\hline Unesp & 3 & USJ & 1 & Ufrgs & 1 \\
\hline UFG & 2 & UEM & 1 & UFSM & 1 \\
\hline $\begin{array}{c}\text { PUC- } \\
\text { Campinas }\end{array}$ & 1 & UEPG & 1 & UFTO & 1 \\
\hline PUCRJ & 1 & UFam & 1 & & \\
\hline PUCRS & 1 & UFBA & 1 & & \\
\hline PUCSP & 1 & Ufes & 1 & & \\
\hline Fo
\end{tabular}

Fonte: dados da pesquisa.

Assim, observamos que, apesar da dispersão das pesquisas entre os programas de pós-graduação, o maior número de publicações sobre o tema concentra-se nos programas de pós-graduação em Educação da Universidade Federal da Paraíba (6) e da Universidade Federal do Ceará (5). A análise preliminar indica a prevalência de estudos sobre o tema nos programas de pós-graduação em educação dessas duas universidades do Nordeste na última década. Além disso, o conjunto de estudos foi desenvolvido predominantemente em universidades públicas ou comunitárias. Essas instituições, ainda hoje, permanecem como os principais centros de pesquisa e formação de mestres e doutores no país.

No quadro 2 descrevemos o título das teses e dissertações e a identificação de seus respectivos autores com 0 ano de publicação. As referências detalhadas podem ser encontradas na bibliografia. 
Quadro 2 -

Lista dos autores e ano das teses selecionadas para esta revisão de literatura.

Albuquerque Santos, 2019; Affonso, 2014; Aguiar, 2009; Albuquerque, 2012; Andrade, 2010; Assis, 2012; Azevedo, 2015; Buoro, 2013; Cardoso, 2012; Carvalho, 2016; Dantas, 2017; Favarão, 2012; Costa, 2010; Guerra, 2009; Guimarães, 2010; Isabel, 2016; Justino, 2013; Lacerda, 2019; Leal, 2014; Leimig, 2012; Melo, 2011; Mendes, 2016; Morais, 2009; Nascimento, 2011; Moro Nascimento, 2012; Oliveira, 2011; Coelho Oliveira, 2015; Prasniski, 2015; Rabelo, 2010; Rocha, 2019; Rodrigues Santos, 2017; Sodré Santos, 2015; Silva, 2014; Soares, 2012; Freitas Soares, 2014; Torres, 2013; Tiné, 2009; Uler, 2010; Vidal, 2019; Villar, 2009.

Fonte: dados da pesquisa.

Após a leitura e exploração textual dos trabalhos identificamos os referenciais teóricos usados nas discussões dos resultados.

Gráfico 1 -

Número de teses e dissertações que citaram cada autor como principal referencial teórico da pesquisa.

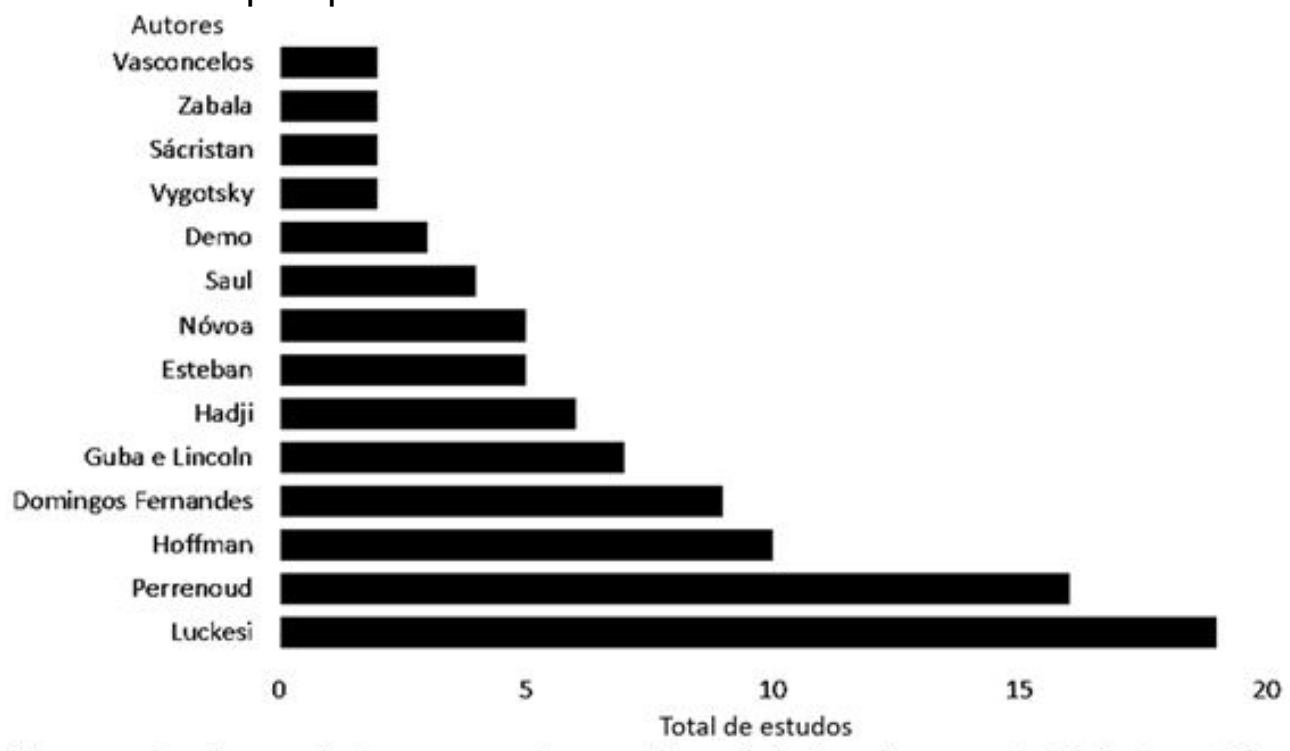

(A soma do número de teses por autores referenciais é maior que o total de teses/dissertações. Os estudos discutem seus dados com mais de um referencial teórico.)

Fonte: dados da pesquisa.

Constatamos que Cipriano Luckesi é o autor mais citado nessas publicações. Luckesi, professor emérito da Universidade Federal da Bahia, é um dos principais pesquisadores que publicam no campo da filosofia da educação no país. Sua obra intelectual é vasta e voltada para o campo da didática e da avaliação da aprendizagem.

Além disso observamos que, dentre os referenciais que fundamentam as 40 pesquisas, predominam teóricos procedentes de países de língua portuguesa. Tal evidência indica um caminho prodigioso diante de novos referenciais a serem explorados por meio do contato com obras ainda não traduzidas para nosso idioma 
Cabe salientar, ainda, que o referencial construtivista da avaliação da aprendizagem é o ponto de convergência entre todos os pesquisadores citados no gráfico 1, embora esses autores não comunguem das mesmas escolas de pensamento. Apesar disso eles são consonantes, em suas obras, com uma perspectiva avaliativa diagnóstica, formativa e com o objetivo de favorecer as aprendizagens para os estudantes. Desse modo podemos deduzir, com base nessas aproximações, que suas ideias pedagógicas se distanciam dos vieses seletivos e classificatórios presentes na pedagogia tradicional.

No que se refere às metodologias utilizadas no universo amostral de teses e dissertações que analisamos encontramos um estudo de revisão de literatura, uma pesquisa documental e outra de estado da arte. Os estudos de revisão apresentam contribuições significativas para os pesquisadores do campo, no sentido de apresentar o estado do conhecimento da área de interesse. Assim, ao descrever as tendências, as formas e os direcionamentos seguidos destacam-se como uma metodologia que oferece um horizonte de partida para outros estudos. Essas investigações, entretanto, permanecem pouco exploradas nesses artigos nos últimos dez anos. Além disso, também identificamos um estudo quantitativo e uma investigação com metodologia mista.

Tabela 2 -

Metodologias de pesquisa utilizadas pelas teses e dissertações analisadas e publicadas no período 2009-2019.

\begin{tabular}{l|c}
\multicolumn{1}{c|}{ Metodologia } & Quantidade \\
\hline Pesquisa qualitativa & 17 \\
\hline Pesquisa qualitativa etnometodológica & 1 \\
\hline Pesquisa qualitativa estudo de caso & 15 \\
\hline Pesquisa qualitativa participativa & 2 \\
\hline Pesquisa quantitativa & 1 \\
\hline Pesquisa mista & 1 \\
\hline Estado da arte & 1 \\
\hline Pesquisa documental & 1
\end{tabular}

Fonte: dados da pesquisa.

Dentre essas publicações predominam pesquisas qualitativas (35), preponderantemente estudos de caso (15). De acordo com André (2006) essas abordagens qualitativas têm ganhado centralidade nas pesquisas em educação e se caracterizam por integrar "um conjunto heterogêneo de métodos, de técnicas e de análises, que vão desde os estudos etnográficos, as pesquisas participantes, os estudos de caso até a pesquisa-ação e as análises de discurso, de narrativas, de histórias de vida" (p. 4).

Dessas metodologias também resultaram amostras qualitativas de participantes dos estudos. 
Figura 3 -

Diagrama de dispersão do número de professores participantes das pesquisas analisadas.

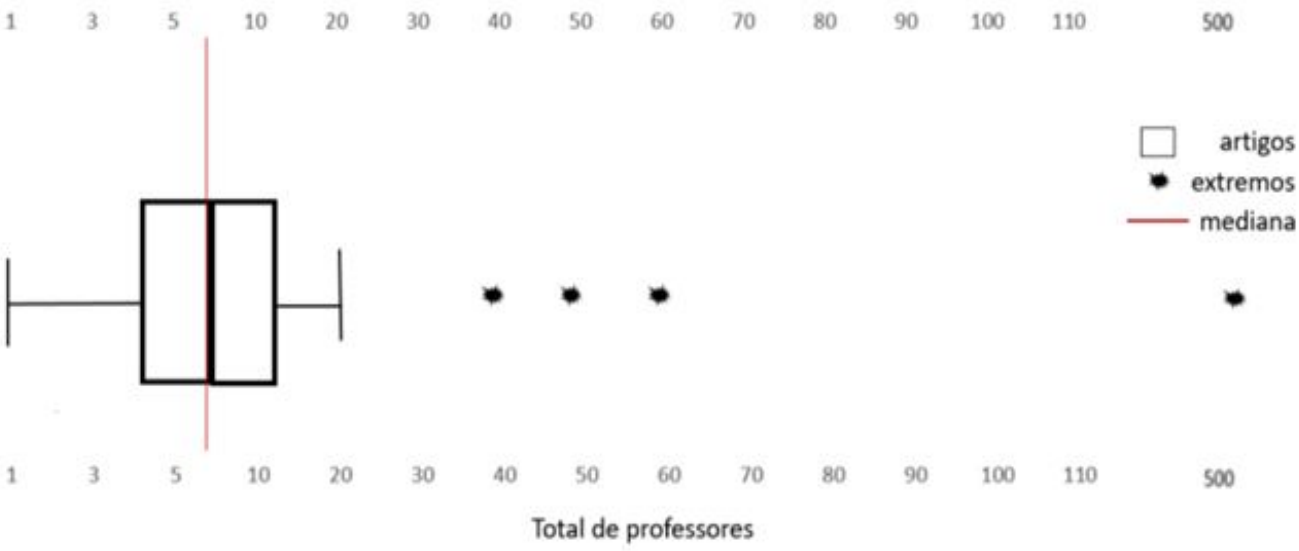

No diagrama, a mediana corresponde a seis participantes [ $1^{\circ}$ quartil: 4 ; mediana: $6 ; 3^{\circ}$ quartil: 12$]$ $50 \%$ dos estudos possuem menos que seis, $25 \%$ envolveram entre seis e doze professores e $15 \%$, entre doze e vinte. Quatro estudos envolveram, respectivamente, 40, 50, 60 e 500 docentes (10\%)

Fonte: dados da pesquisa.

Em consequência disso implicaram a prevalência de investigações com um número de participantes inferior a 12 professores. De acordo com o gráfico, dentre as 40 teses e dissertações, a mediana do total de sujeitos da pesquisa é seis - um reflexo da natureza metodológica mais frequente.

Nesse sentido Ludke e André (1986) descrevem que as pesquisas qualitativas têm o potencial de superar algumas limitações das pesquisas educacionais, visando contribuir para a compreensão das lacunas deixadas pelas análises estatísticas multivariáveis de estudos mais abrangentes. Desse modo as autoras ponderam que seus resultados "possibilitam esclarecer todo o emaranhado de relações de causalidade e de interrelações de um universo particularmente fluido como é o campo educacional" (p. 99). Nesse sentido o estudo de caso expressa relevante capacidade descritiva do campo investigativo em função de suas delimitações analíticas dentro da complexidade escolar.

Pela tabela 3 pode-se observar as formas de coleta de dados utilizados nas 40 pesquisas.

Tabela 3 -

Formas de coleta de dados utilizadas nas 40 teses e dissertações analisadas publicadas no período 2009-20194

\begin{tabular}{l|c}
\multicolumn{1}{c|}{ Metodologia } & Quantidade \\
\hline Entrevistas semiestruturadas & 31 \\
\hline Observação etnometodológica & 14 \\
\hline Análise documental & 13 \\
\hline Entrevistas fechadas & 12 \\
\hline Análise de diários e provas & 6 \\
\hline
\end{tabular}

${ }^{4}$ Trinta e sete estudos utilizaram combinações metodológicas - o total de ferramentas não corresponde ao total de artigos. 


\begin{tabular}{l|l}
\hline Narrativas, memórias escolares & 2 \\
\hline Pesquisa bibliográfica & 2 \\
\hline Grupos focais & 1 \\
\hline
\end{tabular}

Fonte: dados da pesquisa.

Verificamos que a obtenção de material verbal por meio de entrevistas possui maior prevalência em relação às demais formas de coleta de dados. Estas foram realizadas por meio de questionários fechados, entrevistas abertas, grupos focais e narrativas. Ao todo, 37 estudos combinaram dois ou mais métodos na triangulação dos dados, uma estratégia promissora para ampliar o foco de análise de seus objetos.

Assim, o pesquisador tem a possibilidade de contrapor diferentes perspectivas de seu objeto de estudo e, então, pode confrontar ou até mesmo sobrepor seus resultados, aumentando a solidez de suas hipóteses. Observamos uma clara homogeneidade em relação às metodologias e ferramentas de pesquisa utilizadas nas teses e dissertações que compõem o universo amostral deste estudo, além da raridade do uso de alguns métodos e técnica de produção de dados.

Considerando a avaliação da aprendizagem como objeto de investigação das teses e dissertações analisadas, também buscamos caracterizar o perfil de seus participantes.

Tabela 4 -

Perfil dos participantes das pesquisas analisadas publicadas no período 2009-2019.

\begin{tabular}{|c|c|c|}
\hline & Participantes dos estudos & Número de artigos \\
\hline & Professores da educação básica & 1 \\
\hline & Professores do ensino fundamental I & 4 \\
\hline & Todas as disciplinas & 4 \\
\hline $0 \bar{\Phi}$ & Artes & 4 \\
\hline 0 & Ciências & 4 \\
\hline ळ है & Espanhol & 1 \\
\hline 市 & Educação física & 2 \\
\hline $\mathscr{d}$ & Geografia & 1 \\
\hline 흥 & História & 1 \\
\hline $0 \cdot \frac{5}{0}$ & Matemática & 2 \\
\hline ฮิ & Subtotal & 19 \\
\hline & Todas as disciplinas & 7 \\
\hline is $=0$ & Biologia & 2 \\
\hline$\stackrel{\oplus}{\Xi}$ & Inglês & 1 \\
\hline 禹 & Matemática & 1 \\
\hline$\Phi \cdot \frac{5}{\omega}$ & Química & 1 \\
\hline$\stackrel{\circ}{0}$ & Subtotal & 12 \\
\hline Educa & ăo de jovens e adultos & 1 \\
\hline Estud & de revisão de literatura, estado da arte & 3 \\
\hline Total & & 40 \\
\hline
\end{tabular}

Fonte: dados da pesquisa. 
Notamos que o foco investigativo de metade das teses e dissertações são os professores dos anos finais do ensino fundamental, de modo que apenas quatro estudos direcionam seu olhar para os professores dos anos iniciais do ensino fundamental e doze são voltadas para o contexto do ensino médio.

As características que identificamos nas publicações em teses e dissertações sobre o objeto deste estudo são, em sua maioria, de natureza qualitativa, direcionadas ao ensino fundamental, feitas com pequenos grupos amostrais de docentes. Além disso as entrevistas são os meios de produção de dados mais utilizados. Com base nessas características elas revelam um padrão já evidenciado por outros autores para as pesquisas educacionais no campo da avaliação da aprendizagem (Jacomini, 2014; Costa, 2010). Tais características mostram permanências e uma hegemonia em relação aos aspectos desses estudos, já constatadas por Gatti (2001) há duas décadas.

Também acareamos os 40 estudos em relação às especificidades de seus objetivos. Pelo fato deste estudo ter um foco direcional todas as pesquisas selecionadas tratam necessariamente do objeto 'percepções e representações sociais de professores acerca de avaliação'. Em função disso optamos pela especificação de suas particularidades com a finalidade de analisarmos com maior objetividade os resultados encontrados em cada estudo.

Tabela 5 -

Total de artigos selecionados em relação aos objetivos do estudo.

\begin{tabular}{l|c}
\hline Objetivos específicos da pesquisa & Quantidade \\
\hline $\begin{array}{l}\text { Pesquisa ação: investigações sobre o processo avaliativo } \\
\text { de professores em formação continuada }\end{array}$ & 1 \\
\hline $\begin{array}{l}\text { Examinar as práticas e concepções avaliativas de } \\
\text { professores }\end{array}$ & 9 \\
\hline $\begin{array}{l}\text { Revisão bibliográfica, estado da arte com foco nas } \\
\text { percepções e representações sociais de professores } \\
\text { acerca de avaliação da aprendizagem }\end{array}$ & 3 \\
\hline $\begin{array}{l}\text { Conhecer as percepções e representações sociais de } \\
\text { professores acerca de avaliação da aprendizagem }\end{array}$ & 27 \\
\hline
\end{tabular}

Fonte: dados da pesquisa.

Cabe salientar que as categorias de objetivos foram tipificadas com o referencial da leitura exploratória do material textual, o qual permitiu identificarmos seis classes de objetivos. Sucessivamente iniciamos o diálogo com os resultados de pesquisa descritos pelos artigos de acordo com seus objetivos e desfechos apresentados.

Diante desse caminho definido prosseguimos a descrição dos resultados dos estudos referenciados pelos objetivos anteriormente apresentados. Dentre as quarenta pesquisas que compõem nosso conjunto amostral três direcionaram seus esforços para apresentar o panorama mais amplo do campo da avaliação da aprendizagem. Nesse sentido, buscando as perspectivas do estado da arte, Uler (2010) fez um balanço das concepções de avaliação da aprendizagem veiculadas à produção acadêmica em programas de Educação de três universidades - PUCSP, USP, Unicamp - no período de 2000 a 2007. A autora destaca "a prevalência de um tratamento tradicional de avaliação, 
influenciado por um pensamento positivista" e salienta que "a mudança nas práticas escolares ainda está longe de ser desejável (Uler, 2010, p. 136), seja nas escolas seriadas ou organizadas em ciclos.

Nessa perspectiva Costa (2010) investigou as concepções de avaliação da aprendizagem predominantes no pensamento educacional brasileiro no período de 1999 a 2008. A autora descreve que a perspectiva mediadora e qualitativa tem se destacado na literatura, a qual enfatiza a necessidade de investimentos na formação de professores para que se promova a efetivação de uma avaliação formativa nas salas de aula.

A revisão sistemática produzida por Villar (2009), com foco na escola organizada por ciclos (período de 2000 a 2007), aponta que a implantação das políticas dessa forma de organização escolar não promoveu mudanças significativas nas práticas de avaliação dos professores. Diante de tal perspectiva, para o período analisado, os três estudos indicam que as práxis docentes tendem a ser classificatórias, somativas e tradicionais, mesmo em contextos nos quais a seriação não condiciona a progressão dos estudantes entre as séries.

A pesquisa-ação de Mendes (2016), realizada com professores de educação física em formação continuada, destaca especificidades do habitus dos professores dessa disciplina escolar. A autora afirma que, no início da carreira, os docentes reproduzem as práticas avaliativas vivenciadas em sua vida escolar. Contudo, sua pesquisa descreve que há uma tendência de mudanças para um paradigma avaliativo diagnóstico e formativo ao longo do desenvolvimento profissional desses professores. Tal processo é influenciado pelas exigências da legislação e pelos objetivos contemporâneos do campo disciplinar.

No conjunto amostral de teses e dissertações identificamos nove pesquisas que relacionaram as percepções de professores e suas práticas avaliativas. $\mathrm{Na}$ análise comparativa dos resultados, consideramos fundamental estarmos atentos às particularidades de cada disciplina e cada segmento educacional.

No contexto do ensino fundamental II Melo (2011) evidenciou que a participação e o envolvimento dos alunos nas aulas de educação física são importantes elementos norteadores nos processos avaliativos feitos pelos professores, portanto, prevalecem em relação à medição e quantificação de resultados de desempenhos físicos nesse estudo de caso. Esses resultados divergem daqueles encontrados nas demais pesquisas que também triangularam as percepções dos docentes com a observação de sua práxis.

Quando juntamos oito pesquisas ${ }^{5}$ realizadas com professores de diferentes disciplinas e segmentos da educação básica, percebemos que seus resultados se confluem ao apontarem a predominância de práticas de classificação e seleção dos estudantes, influenciando as concepções e formas de avaliar dos professores. Esses estudos também especificam a configuração de tensões entre as concepções dos docentes e suas respectivas ações pedagógicas e, apesar de apresentarem indícios do reconhecimento da necessidade de práticas avaliativas contínuas formativas, relatam que a estrutura escolar exerce forte influência sobre suas formas de avaliação.

5 Tiné, 2009; Leal, 2014; Affonso, 2014; Silva, 2014; Prasniski, 2015; Oliveira, 2015; Isabel, 2016; Coelho Dantas, 2017. 
Nesse sentido categorizamos 27 teses e dissertações que investigaram, nesses termos, as representações e percepções de professores sobre avaliação. Nesse grupo quatro pesquisas ${ }^{6}$ foram direcionadas para os docentes das séries iniciais do ensino fundamental e as quatro análises indicam que as percepções dos professores manifestam coerência e proximidade com o paradigma da avaliação formativa, ou seja, aquela cujo "propósito mais fundamental é apoiar as aprendizagens dos alunos [...], e, assim sendo, é entendida como um processo eminentemente pedagógico e intrinsecamente relacionado com as aprendizagens dos alunos" (Fernandes, 2019, p. 12). Entretanto, os estudos retratam que as representações dos docentes são híbridas: seus discursos repetem os ideais educacionais construtivistas e contemporâneos. Todavia, de acordo com essas pesquisas, essas concepções pouco orientam suas práticas.

Dentre os estudos relacionados diretamente a percepções e representações dos professores quatorze exploraram o universo de docentes do segundo segmento do ensino fundamental. Em pesquisas direcionadas à escola organizada por ciclos, Soares (2012) e Buoro (2013) identificaram o distanciamento entre as concepções dos professores e a avaliação classificatória. No entanto, as duas autoras destacaram, nesse contexto, a permanência de práticas historicamente consolidadas na escolarização seriada. De acordo com os dois estudos a proposta de uma estrutura de progressão continuada e cíclica se desenvolve como um modelo híbrido seriado.

Três estudos ${ }^{7}$ abordaram as representações de professores de artes sobre avaliação. Nos três casos os autores também destacam o entrelaçamento entre as percepções de uma avaliação mediadora e as dificuldades de corporificá-las em suas práticas escolares. Nos outros estudos com professores do ensino fundamental II de diferentes disciplinas (9), os autores ${ }^{8}$ também destacam que prevalecem crenças classificatórias de avaliação entre os docentes. As pesquisas ressaltam que suas concepções expressam a necessidade de um processo avaliativo que colabore, de fato, para as aprendizagens dos estudantes.

Nas pesquisas direcionadas a professores do ensino médio, quase todos os autores ${ }^{9}$ salientaram a centralidade de uma cultura de exame nas práticas dos professores. Essas análises revelam fragilidades quanto às concepções sobre avaliação da aprendizagem dos docentes, embora eles declarem reconhecer a necessidade de se apropriarem de novas perspectivas pedagógicas para seu processo de profissionalização. Cabe especificar, ainda, que os estudos expõem fatores limitadores relacionados, principalmente, à formação docente e aos obstáculos da estrutura escolar.

Esses apontamentos vão de encontro às considerações de Gatti (2016) sobre a necessidade de investimentos na qualidade da formação de professores e nas condições críticas do trabalho docente. De acordo com a autora esse problema pode ser mais bem

${ }^{6}$ Guerra, 2009; Aguiar, 2012; Torres, 2013; Vidal, 2019.

7 Assis, 2012; Guimarães, 2010; Justino, 2013.

8 Rabelo, 2010; Nascimento, 2011; Albuquerque, 2012; Cardoso Oliveira, 2012; Favarão, 2012; Oliveira, 2012; Azevedo, 2015; Santos, 2017; Rocha, 2019.

9 Morais, 2009; Andrade, 2010; Leiming, 2012; Moro Nascimento, 2012; Costa, 2010; Freitas Soares, 2014; Sodré Santos, 2015; Carvalho, 2016; Albuquerque Santos, 2019; Lacerda, 2019.

Regae: Rev. Gest. Aval. Educ.

Santa Maria

v. 10

n. 19

e55396, p. 1-21

2021 
compreendido com o suporte de pesquisas que buscam descrever as questões relacionadas à dinâmica e à complexidade da identidade profissional da categoria e indicar caminhos para superar seus dilemas.

Configuram-se, por esse motivo, pertinentes investigações voltadas para o campo da avaliação. Esse terreno, de acordo com os resultados majoritários das teses e dissertações analisadas no presente estudo, ainda se revela cercado por concepções classificatórias que povoam as representações sociais de professores, limitando seu potencial pedagógico no contexto da escolarização. Por essa razão,

têm, há muitas décadas, impregnado o sistema educacional, com fundamentos que justificam a reprovação dos alunos na educação escolar a que têm direito. É urgente, portanto, provocar junto a educadores, famílias e sociedade em geral, uma substancial reflexão, no sentido de substituir a repetência e a evasão por uma avaliação que promova o aperfeiçoamento contínuo das crianças e dos jovens. (Firme, 1994, p. 1)

Por conta desses fatores conhecer o conteúdo do pensamento social e trazê-lo para o debate é fundamental para as discussões em torno da formação de professores, da prática docente e da escolarização desejada por cada contexto educacional.

\section{Considerações finais}

Apesar do presente estudo ter se desenvolvido com o objetivo de compreender o conhecimento recente produzido pela literatura dedicada ao campo da avaliação da aprendizagem e seus aspectos relacionados às dimensões das representações sociais de professores da educação básica, ele almejou também abranger a diversidade de abordagens teóricas, de métodos investigativos e analíticos, bem como suas possibilidades e limitações enquanto ferramentas de pesquisa.

A análise teve como alvo as bases de dissertações e teses disponibilizadas na plataforma BDTD publicados no período entre 2009 a 2019. Na elaboração do trabalho buscou-se o referencial de estudos de revisão relacionados à mesma temática desenvolvidos em períodos anteriores, a fim de estabelecer uma continuidade com o estado do conhecimento do campo.

Nesse sentido, o foco da discussão centrou-se nas investigações relacionadas às representações sociais de professores acerca de avaliação. Essas representações são teorias construídas pelos sujeitos dos grupos sociais para operar o funcionamento de sua realidade e esse conjunto de saberes socialmente compartilhado pode ser estudado a partir das percepções, das concepções, das atitudes, das associações, das observações e outras manifestações dos sujeitos que pertencem ao grupo, segundo Jodelet (2001). 0 conhecimento desses indivíduos é relevante nas pesquisas educacionais, já que as representações dos professores influenciam sua atuação pedagógica. Com base em tal perspectiva o referido objeto compõe um conteúdo importante para a compreensão dos significados e sentidos relacionados aos dilemas e desafios da profissão docente na direção de sua superação, de acordo com Mazzotti (1994). 
As pesquisas selecionadas analisaram crenças, opiniões, concepções, vivências e práticas de professores em diferentes contextos educacionais. Entretanto, ressaltamos que, mesmo que esses estudos tenham explorado as múltiplas dimensões do pensamento social de professores sobre avaliação, ainda são escassos os trabalhos desenvolvidos sob a perspectiva teórica e metodológica das representações sociais.

Em verdade elas descrevem a complexidade da práxis avaliativa como um desafio para a profissão docente. Essa diversidade de pesquisas qualitativas, por sua vez, revela as particularidades dos seus grupos amostrais, mas, como salienta Luckesi (2002), também apresenta um conjunto de representações há muito tempo predominante nos grupos sociais que vivenciam o magistério.

Diante do cenário descrito pelo universo amostral deste estudo há indicações de que as representações sociais dos docentes da educação básica, no que diz respeito à avaliação, são influenciadas pela permanência de uma mentalidade tradicional que associa essa prática ao que Luckesi (2014) chama de 'pedagogia do exame'. Observa-se que o contexto e a normatividade das instituições escolares onde lecionam são fatores presentes na complexidade que envolvem as representações de avaliação dos professores. Todavia, reconhecemos que são necessárias investigações direcionadas ao papel dos processos de formação e profissionalização docente em seu habitus profissional avaliativo para ampliar a profundidade da discussão.

Desse modo, mesmo em contextos educacionais de progressão continuada e cíclica, nos quais os processos avaliativos não são diretamente condicionados à promoção e à seriação, os estudos observam que o pensamento de avaliação associado à classificação dos estudantes é manifestado pelos professores. Apesar disso, muitos desses estudos demonstram um hibridismo entre as concepções tradicionais e construtivistas no pensamento dos professores participantes.

Diante de tal perspectiva evidencia-se que há permanências nas mudanças do pensamento educacional, indicando que a incorporação das ideias de uma avaliação voltada de fato para favorecer a aprendizagem não ocorre de forma abrupta. Pelo contrário, é lentamente que essas perspectivas vão sendo incorporadas às práticas sociais já estabelecidas na práxis docente. Em geral as investigações revelam que os professores têm conhecimento teórico de uma avaliação formativa e mediadora, mas reconhecem dificuldades de incorporar tais saberes em seu ofício profissional. Essa constatação também é ratificada pelas pesquisas direcionadas à observação do cotidiano escolar.

Apesar de os estudos apresentarem muitas convergências seus resultados também traduzem as particularidades de cada contexto educacional. As pesquisas direcionadas à disciplina de educação física, por exemplo, indicam singularidades não evidenciadas por aquelas dirigidas para outros campos disciplinares. Por conta disso esses resultados reforçam o papel das pesquisas qualitativas e dos estudos de caso como uma lente analítica muito relevante para a compreensão da complexidade das questões educacionais.

Sendo assim, identificamos três pesquisas que revisaram o estado da arte e a literatura da grande área da avaliação em um período que compreende mais de duas décadas (1999-2014). Os autores desses estudos destacaram que na literatura e no debate acadêmico houve a expansão de uma perspectiva da avaliação como uma

\begin{tabular}{|l|l|l|l|l|l|}
\hline Regae: Rev. Gest. Aval. Educ. & Santa Maria & v. 10 & n. 19 & e55396, p. 1-21 & 2021 \\
\hline
\end{tabular}


"construção social complexa, um processo intrinsecamente pedagógico e didático, plenamente integrado no ensino e na aprendizagem, deliberado e interativo, cuja principal função é regular e melhorar as aprendizagens dos alunos" (Fernandes, 2008, p. 357). Apesar das mudanças as pesquisas apreciadas por esses estudos de revisão também sinalizaram que essas concepções não estão sendo incorporadas na mesma velocidade na práxis pedagógica dos professores da educação básica.

Desse modo, essas análises reforçam os resultados apontados pelos estudos de caso que selecionamos. Os resultados também se afinam com as considerações de Calderón e Poltronieri (2013) acerca das pesquisas em estado da arte no campo da avaliação (1980-2007), as quais traduzem um abismo entre os referenciais teóricos de uma pedagogia emancipatória no meio acadêmico e as escolas. Assim, eles retratam que prevalecem práticas pedagógicas tradicionais, classificatórias e certificativas nas escolas. Apesar disso, a hegemonia de uma literatura voltada para uma avaliação formativa e emancipatória, e seu reflexo nas representações dos professores, as quais transparecem seu conhecimento e suas limitações por meio de elementos contraditórios do discurso, enunciam um processo de mudança de pensamento social.

De acordo com Moscovici (2009) essas transformações estão relacionadas à necessidade de ajustar à sua realidade aquilo que não é familiar, incorporando ou rejeitando esses elementos em seu mundo. É nessa dinâmica que as representações sociais são construídas pelos sujeitos de um grupo, pois dão sentido aos fenômenos e orientam como nos comportar em relação a ele. Em função disso, esse é um conhecimento culturalmente e historicamente construído pelos agentes que se relacionam, influenciando suas práticas cotidianas.

Desse modo, entender esses mecanismos que envolvem a construção de sentidos nos grupos sociais de docentes é muito significativo na busca de soluções para os problemas educacionais. A escolarização, que atualmente enfrenta desafios relacionados aos seus papéis na contemporaneidade, também é um espaço que se depara com ressignificações da sua cultura e práticas frente às questões das novas gerações.

Por esse motivo, compreender o pensamento social dos grupos de professores em relação a temas como a avaliação pode trazer contribuições positivas às reflexões sobre as políticas educacionais, a estrutura escolar, a formação inicial, a profissionalização docente e, principalmente, sobre os potenciais pedagógicos da prática avaliativa, a qual é uma poderosa ação para auxiliar os estudantes a melhorar suas aprendizagens.

\section{Referências}

AFFONSO, Dalva Mariana. Concepções e práticas de professores de ciências sobre avaliação da aprendizagem. São Paulo: Unesp, 2010. 118f. Tese (Doutorado em Educação). Universidade Estadual Paulista, 2014.

AGUIAR, Elenita de Sousa. Concepções e práticas de professores em avaliação da aprendizagem na rede pública municipal de ensino de Teresina. Fortaleza UFC, 2009. 119f. Dissertação (Mestrado em Educação). Universidade Federal do Ceará.

ALBUQUERQUE, Leila Cunha de. Avaliação da aprendizagem: concepções e práticas do professor de matemática dos anos finais do ensino fundamental. Brasília UNB, 2012. 165f. Dissertação (Mestrado em Educação). Universidade de Brasília. 
ALBUQUERQUE SANTOS, Vilma de. Avaliação da aprendizagem na perspectiva dos professores que lecionam a disciplina de química no ensino médio integrado do IFPE. Recife: UFPE, 2019. 107f. Dissertação (Mestrado em Educação). Universidade Federal de Pernambuco.

ANDRADE, José Alexandre Leite de. Avaliação da aprendizagem: do discurso revelado à prática pedagógica: um estudo de caso numa escola de ensino médio em Maracanaú/CE. Fortaleza: UFCE, 2010. 173f. Dissertação (Mestrado em Educação). Universidade Federal do Ceará.

ANDRÉ, Marli Eliza Dalmazo Afonso. Pesquisa em educação: desafios contemporâneos. Pesquisa em educação ambiental, São Paulo, v. 1, n. 1, 2006, p. 43-57.

ASSIS, Thiago Santos de. Avaliação da aprendizagem em dança: um trânsito entre o dito e o feito em escolas municipais de Salvador. Salvador: UFBA, 2012. 123f. Dissertação (Mestrado em Dança). Universidade Federal da Bahia.

AZEVEDO, S. C. A construção discursiva de posicionamentos sobre avaliação educacional: um estudo sistêmico-funcional com professores da educação básica. Rio de Janeiro: PUC-Rio, 2010. 228f. Tese (Doutorado em Educação). Pontifícia Universidade Católica do Rio de Janeiro.

BARDIN, Laurence. Análise de conteúdo. São Paulo: Edições 70, 2011.

BUORO, Edna et al. Avaliação da aprendizagem nos ciclos e na progressão continuada a partir das concepções de atores do processo educacional. Campinas: PUC-Campinas, 2013.153f. Dissertação (Mestrado em Educação) Pontifícia Universidade Católica de Campinas.

CALDERÓN, Adolfo Ignacio. POLTRONIERI, Heloisa. Avaliação da aprendizagem na educação básica: as pesquisas do estado da arte em questão (1980-2007). Revista Diálogo Educacional, Curitiba, v. 13, n. 40, 2013, p. 873-893.

CARVALHO, Wirla Risany Lima. Tessituras da formação em avaliação educacional: os desafios à cultura de avaliação e a busca por uma educação crítico-reflexiva. Fortaleza: UFCE, 2016. 121f. Tese (Doutorado em Educação). Universidade Federal do Ceará.

COELHO OLIVEIRA, Sandra Maria. A avaliação formativa como regulação de aprendizagem: desafios para a práxis no ensino médio da rede pública estadual do Ceará em uma análise fenomenológica. Fortaleza: UFCE, 2010. 257f. Tese (Doutorado em Educação). Universidade Federal do Ceará.

COSTA, Simone Freitas Pereira. Concepções de avaliação da aprendizagem: um balanço de produções no período de 1999 a 2008.Goiânia: UCGO, 2010. 170f. Tese (Doutorado em Educação). Universidade Católica de Goiás.

CRESWELL, John W. Projeto de pesquisa métodos qualitativo, quantitativo e misto. Porto Alegre: Artmed, 2007.

DANTAS, Claudio Rejane da Silva. Avaliação no ensino de ciências no nível fundamental: investigando orientações oficiais e práticas docentes, fazendo "escuta" e intervenções em escolas. Porto Alegre: UFRGS, 2017. 443f. Tese (Doutorado em Educação). Universidade Federal do Rio Grande do Sul.

FAVARÃO, Cláudia Fátima de Melo. Avaliação da aprendizagem: concepções e características. Londrina: UEL, 2012. 95f. Dissertação (Mestrado em Educação). Universidade Estadual de Londrina. 
FERNANDES, Domingos. Para uma teoria da avaliação no domínio das aprendizagens. Estudos em avaliação educacional, São Paulo, v. 19, n. 41, 2008, p. 347372.

FERNANDES, Domingos. Para um enquadramento teórico da avaliação formativa e da avaliação sumativa das aprendizagens escolares. In: ORTIGÃO, Maria Isabel Ramalho; FERNANDES, Domigos; PEREIRA, Talita Vidal; SANTOS, Leonor (orgs). Avaliar para aprender em Portugal e no Brasil: Perspectivas teóricas, práticas e de desenvolvimento. Curitiba: CRV, 2019, p. 139-164.

FIRME, Thereza Penna. Mitos na avaliação: diz-se que... Revista Meta: Avaliação, v. 1, n. 1, 2009, p. 1-10.

FLICK, Uwe. Introdução à pesquisa qualitativa. Porto Alegre: Artmed, 2009.

GATTI, Bernadete. Implicações e perspectivas da pesquisa educacional no Brasil contemporâneo. Cadernos de pesquisa, São Paulo, n. 113, 2001, p. 65-81.

GATTI, Bernadete. Formação de professores: condições e problemas atuais. Revista internacional de formação de professores, São Paulo,v. 1, n. 2, 2016, p. 161-171.

GOUGH, David; OLIVER, Sandy; THOMAS, James (ed.). An introduction to systematic reviews. London: Sage, 2017.

GOUGH, David, Systematic Research Synthesis, in Gary Thomas e Richard Pring, eds., Public discussion on the meaning, value and use of an evidence-based approach in higher education. New York: Open University Press, 2004. p. 44-62.

GUERRA, Gleice Kelly de Souza. Avaliação processual: um estudo das representações sociais de professoras da rede municipal de ensino do Recife. Recife: UFPE, 2009. 320f. Dissertação (Mestrado em Educação). Universidade Federal de Pernambuco.

GUIMARÃES, Ana Luiza B. A Avaliação da aprendizagem em arte: desvelando realidades. Londrina: UEL, 2010. 108f. Dissertação (Mestrado em Educação). Universidade Estadual de Londrina.

ISABEL, Miguel Boa. Avaliação da aprendizagem dos alunos do ensino secundário em Cabinda/Angola. Belo Horizonte: UFMG, 2016. 227f. Tese (Doutorado em Educação). Universidade Federal de Minas Gerais.

JACOMINI, Márcia Aparecida. Avaliação da aprendizagem em tempos de progressão continuada: o que mudou? Um estudo de teses e dissertações sobre o tema (2000-2010). Ensaio: Avaliação e Políticas Públicas em Educação, Rio de Janeiro, v. 22, n. 84, 2014, p. 807-828.

JODELET, Denise. Representações sociais: um domínio em expansão. In Jodelet, Denise (ed). As representações sociais, Rio de Janeiro, Eduerj, 2001, p. 17-44.

JUSTINO, Ana Inez Mottim et al. Concepções e práticas de avaliação da aprendizagem de professores de artes visuais. Ponta Grossa: UEPG, 2013. 156f. Dissertação (Mestrado em Educação). Universidade Estadual de Ponta Grossa.

LACERDA, Divaniella de Oliveira. Avaliação da aprendizagem no ensino de Biologia: concepções e indicativos da prática docente. João Pessoa: UFPB, 2019. $131 \mathrm{f}$. Dissertação (Mestrado em Educação). Universidade Federal da Paraíba.

LEAL, Maria Lindalva Gomes. Concepções e práticas de avaliação do ensinoaprendizagem: foco no Proeja. Fortaleza: UFCE, 2014. 126f. Dissertação (Mestrado em Educação). Universidade Federal do Ceará. 
LEIMIG, Eveline Silva de Freitas. Avaliação da aprendizagem de inglês como língua estrangeira: um estudo sobre as concepções de professores de escolas de referência em ensino médio integral em Pernambuco. Recife: UFPE, 2012. 158f. Dissertação (Mestrado em Educação), Universidade Federal de Pernambuco.

LUCKESI, Cipriano Carlos. Avaliação da aprendizagem na escola e a questão das representações sociais. Eccos Revista Científica, São Paulo, v. 4, n. 2, 2002, p. 79-88.

LUCKESI, Cipriano Carlos. Avaliação da aprendizagem escolar: estudos e proposições. São Paulo: Cortez, 2014.

LUCKESI, Cipriano Carlos. Avaliação em educação: questões epistemológicas e práticas. Salvador: Cortez, 2018.

LUDKE, Menga; ANDRÉ, Marli E. D. A. Pesquisa em educação: abordagens qualitativas. São Paulo: Pedagógica e Universitária, 1986.

MAZZOTTI, Alda. Representações sociais: aspectos teóricos e aplicações à educação. Em aberto, Brasília, v. 14, n. 61, 1994, p. 60-78.

MELO, Luciene Farias. Tecendo tramas sobre avaliação da aprendizagem em aulas de educação física escolar. São Paulo: USJT, 2011. 332f. Dissertação de Mestrado. Universidade São Judas Tadeu.

MENDES, Evandra Hein. Avaliação da aprendizagem na educação física escolar: caracterização e habitus de professores do Paraná. Maringá: UEM, 2016. 127f. Tese (Doutorado em Educação Física). Universidade Estadual de Maringá.

MORAIS, Ruth Longuinho de et al. Do discurso à prática: como se caracteriza a avaliação da aprendizagem escolar entre professores de biologia do ensino médio. Goiânia: UFG, 2009. 132f. Dissertação (Mestrado em Educação). Universidade Federal de Goiás.

MOSCOVICl, Serge. Representações sociais: investigações em psicologia social. Petrópolis: Vozes, 2009.

NASCIMENTO, Claudenice Maria Véras. Experienciando a avaliação da aprendizagem na educação de jovens e adultos. Vitória: Ufes, 2009. 184f. Dissertação (Mestrado em Educação). Universidade Federal do Espírito Santo.

NASCIMENTO, Mari Clair Moro. Avaliação da aprendizagem: repercussões de modelos pedagógicos nas concepções docentes. Londrina: UEL, 2012. 125f. Tese (Doutorado em Educação). Universidade Estadual de Londrina.

OLIVEIRA, Décio Luciano Squarcieri. Professores de História dialogam sobre a avaliação da aprendizagem e formação: abordagem (auto)biográfica. Santa Maria: UFSM, 2009. 172f. Dissertação (Mestrado em Educação). Universidade Federal de Santa Maria.

OLIVEIRA, Nelson Roberto Cardoso. Avaliação em Matemática: uma discussão sobre as concepções e práticas de professores do ensino fundamental II da cidade de Campina Grande. Campina Grande UEPB, 2012. 181f. Dissertação (Mestrado em Educação) Universidade Estadual da Paraíba.

PRASNISKI, Maria Elena Tobolski. Avaliação no ensino de ciências: estudo na implantação da pedagogia de projetos em escola pública de ensino básico da região metropolitana de Porto Alegre. Porto Alegre: PUCRS, 2015. 120f. Tese (Doutorado em Educação). Pontifícia Universidade Católica do Rio Grande do Sul.

RABELO, Kamila Santos de Paula. Ensino de geografia e avaliação formativa da aprendizagem: experiências e princípios na rede pública de Goiânia/GO. Goiânia: UFG, 2009. 150f. Dissertação (Mestrado em Geografia). Universidade Federal de Goiás. 
ROCHA, Mariana Rabelo. Crenças de professores de espanhol sobre a avaliação no ensino-aprendizagem de LE. Manaus: Ufam, 2019. 107f. Dissertação (Mestrado em Letras). Universidade Federal do Amazonas.

SANTOS, Nívia Maria. Avaliação da aprendizagem na perspectiva de um grupo de professores da disciplina de Ciências: uma análise do processo em uma escola pública de Cubati/PB. Campina Grande: UEPB, 2017. 128f. Dissertação (Mestrado em Ensino de Ciências e Educação Matemática). Universidade Estadual da Paraíba.

SILVA, Fabio Santana. Práticas avaliativas em arte. João Pessoa: UFPB, 2014. $164 f$. Dissertação (Mestrado em Artes Visuais). Universidade Federal da Paraíba.

SOARES, Fernanda Luzia Sousa Santos. Ciclos de aprendizagem em São Luís: implicações nas práticas avaliativas. São Luís: UFPE, 2012. 156f. Dissertação (Mestrado em Educação). Universidade Federal do Maranhão.

SOARES, Reijane de Freitas. A prática educativa do professor na escola de tempo integral da rede estadual de Teresina-PI. Teresina: UFPI, 2013. 310f. Tese (Doutorado em Educação). Universidade Federal do Piauí.

SODRÉ SANTOS, Valdir. Percepções de docentes de matemática de ensino médio em relação ao processo de avaliação da aprendizagem. Brasília: UNB, 2015. $171 \mathrm{f}$. Dissertação (Mestrado em Educação). Universidade de Brasília.

TINÉ, Sandra Zita Silva. O processo avaliativo em uma escola de ensino médio do Distrito Federal. Brasília: UNB, 2009. 354f. Tese (Doutorado em Educação). Universidade de Brasília.

TORRES, Denise Xavier. Concepções de avaliação da aprendizagem de professoras que atuam em escolas situadas em áreas rurais. Recife: UFPE, 2013. 112f. Dissertação (Mestrado em Educação). Universidade Federal de Pernambuco.

ULER, Arnilde Marta. Avaliação da aprendizagem: um estudo sobre a produção acadêmica dos Programas de Pós-Graduação em Educação (PUC-SP, USP, Unicamp). São Paulo: PUC-SP, 2010. 238f. Tese (Doutorado em Educação). Pontifícia Universidade Católica de São Paulo.

VIDAL, Rita de Cássia Castro. Fazeres docentes e avaliação: um estudo de caso à luz da complexidade. Tocantins: UFT, 2019. 172f. Dissertação (Mestrado em Educação). Universidade Federal do Tocantins.

VILLAR, Ana Paula Russo. A prática avaliativa em uma organização escolar por ciclos de aprendizagem. Recife: UFPE, 2009. 257f. Dissertação (Mestrado em Educação), Universidade Federal de Pernambuco.

Diego Mota é estudante no curso de Doutorado no Programa de Pós-Graduação em Educação da PUC-Rio e professor no Colégio Pedro II.

Orcid: https://orcid.org/0000-0001-5279-8630.

Endereço: Colégio Pedro II - Campo de São Cristóvão, 177 - 20921-903 - Rio de Janeiro - RJ - Brasil.

E-mail: diegoomota@gmail.com. 
Maria Apparecida Campos Mamede-Neves é professora emérita na Pontifícia Universidade Católica do Rio de Janeiro.

Orcid: https://orcid.org/0000-0003-1736-5252.

Endereço: Praça Avenida Heleno Claudio Fragoso, 04/504 - 22793-078 - Rio de Janeiro - RJ - Brasil.

E-mail: apmamede@gmail.com.

Recebido em 28 de setembro de 2020.

Aceito de 19 de novembro de 2020.

(c) (1) $\Theta(\Theta$ 